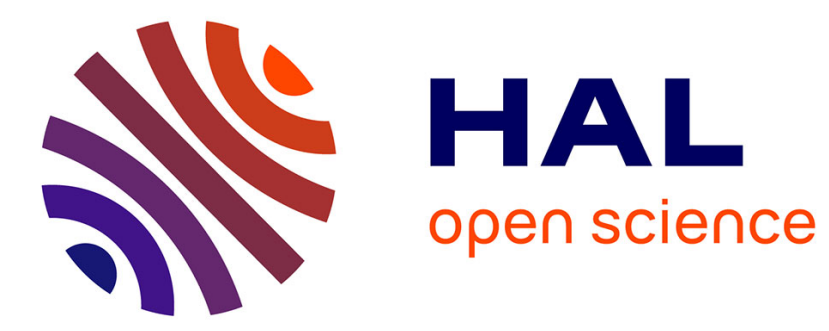

\title{
A spectral envelope approach towards effective SVM-RFE on infrared data
}

\author{
F.E. Spetale, P. Bulacio, S. Guillaume, J. Murillo, E. Tapia
}

\section{To cite this version:}

F.E. Spetale, P. Bulacio, S. Guillaume, J. Murillo, E. Tapia. A spectral envelope approach towards effective SVM-RFE on infrared data. Pattern Recognition Letters, 2016, 71, pp.59-65. 10.1016/j.patrec.2015.12.007 . hal-01728931

\section{HAL Id: hal-01728931 \\ https://hal.science/hal-01728931}

Submitted on 12 Mar 2018

HAL is a multi-disciplinary open access archive for the deposit and dissemination of scientific research documents, whether they are published or not. The documents may come from teaching and research institutions in France or abroad, or from public or private research centers.
L'archive ouverte pluridisciplinaire HAL, est destinée au dépôt et à la diffusion de documents scientifiques de niveau recherche, publiés ou non, émanant des établissements d'enseignement et de recherche français ou étrangers, des laboratoires publics ou privés. 


\title{
A spectral envelope approach towards effective SVM-RFE on infrared data
}

\author{
F. E. Spetale, P. Bulacio, S. Guillaume, J. Murillo, E. Tapia
}

30 October 2015

\begin{abstract}
Infrared spectroscopy data is characterized by the presence of a huge number of variables. Applications of infrared spectroscopy in the midinfrared (MIR) and near-infrared (NIR) bands are of widespread use in many fields. To effectively handle this type of data, suitable dimensionality reduction methods are required. In this paper, a dimensionality reduction method designed to enable effective Support Vector Machine Recursive Feature Elimination (SVM-RFE) on NIR/MIR datasets is presented. The method exploits the information content at peaks of the spectral envelope functions which characterize NIR/MIR spectra datasets. Experimental evaluation across different NIR/MIR application domains shows that the proposed method is useful for the induction of compact and accurate SVM classifiers for qualitative NIR/MIR applications involving stringent interpretability or time processing requirements.
\end{abstract}

\section{Introduction}

Infrared (IR) spectroscopy is a non-invasive technique allowing the identification and characterization of chemical compounds using their interaction with light. Applications of IR spectroscopy in the mid-infrared (MIR) and near-infrared (NIR) bands are of widespread use in many fields, including agriculture [1, 2], food and wines quality $[3,4,5]$, postharvest handling of fruits and vegetables $[6,7]$ and plastic recycling [8].

Main advantages and limitations of MIR and NIR techniques can be explained by the differences in the origin of their absorption spectra. While the MIR spectra follow from the vibration of fundamental bands, the NIR spectra follow from the overtone and combination of fundamental MIR bands. Hence, while the MIR spectra tend to be simple with very sharp and specific peaks, the NIR spectra tend to be rather complex with many broad overlapping bands. Thus, the interpretation of NIR spectra can be very challenging, especially for complex mixtures of samples. However, since the absorption of light in the NIR region $(780-2500 \mathrm{~nm})$ is less intense than in the MIR one $(2500-15000 \mathrm{~nm})$, a deeper penetration of light into matter can be accomplished and a minimal sample preparation is required for NIR applications. 
In practice, IR spectra are presented as high dimensional vectors of factors. For the NIR case, factors are highly correlated. To effectively handle this type of data, dimensionality reduction methods are required. For quantitative applications, with main focus on predictive modeling and not on the identification of relations between factors, Partial Least Squares (PLS) regression methods [9] are traditionally used. Briefly, by means of PLS regression methods, a handy number of latent factors accounting for most of the variation of target responses are first selected and then used to perform linear predictions. On the other hand, for qualitative applications, with main focus just on the identification of robust classification boundaries [10], PLS-DA [11, 12] methods can be applied. However, when interpretability is also required feature selection methods, allowing the identification of relevant classification factors, must be used [13]. This is especially true for almost real-time qualitative NIR applications based on Support Vector Machines (SVM) classifiers [14], a class of machine learning algorithms characterized by their high accuracy and its ability for modeling diverse types of high dimensional data [15]. Applications of SVMs can be found in multiple fields, including bioinformatics [16], sound analysis [17] and chemometrics [18]. Owing to the natural ability of SVMs classifiers to deal with high dimensional data, initial works with SVMs in chemometrics focused more on model selection than on data interpretation or time-processing issues [19, 20], i.e., the complete spectrum of IR datasets were usually considered. However, to accomplish compact and thus interpretable SVM classifiers for almost realtime qualitative applications, a reduced fraction of the IR spectra is required. From the application point of view, working with specific regions instead of the complete spectrum would allow the utilization of IR sensors of higher resolution. To this aim, we first note that the highly correlated nature of the NIR spectra limits the effectiveness of fast univariate feature selection methods assuming the independence between features [21]. Actually, to avoid the selection of redundant features that may be induced by univariate methods, multivariate feature selection, able to take into account interaction between features are recommended. We note, however, multivariate feature selection methods dismiss specific learning aspects of classification methods, a critical aspect in the construction of compact and accurate SVM classifiers.

To introduce specific learning aspects of classification methods into feature selection tasks, embedded feature selection methods are required. For SVM classifiers this can be accomplished with the SVM recursive feature elimination (SVM-RFE) [22] method, a feature selection method built upon SVM classifiers aiming to identify relevant feature subsets. We note, however, that few studies have considered the direct application of SVM-RFE to the problem of NIR samples classification. As mentioned in [23], SVM-RFE can be too computationally expensive, specially when only one least useful feature is removed at each iteration step. Also, SVM-RFE may be unstable with respect to variations in the training data [24]. Although of both these problems may be mitigated with SVM-RFE ensemble variants [25], we note that SVM-RFE does not specifically consider the redundancy between features [26]. Hence, SVM-RFE on IR datasets may lead to the selection of redundant wavelengths and this undesir- 
able effect may be just reinforced by SVM-RFE ensemble variants. Since direct application of SVM-RFE to IR datasets may be suboptimal, alternative feature selection methods based on genetic algorithms [27, 28] and random forest classifiers with PCA [29] have been reported in literature. These considerations strongly suggest that further processing to IR datasets is required before effective SVM-RFE can be accomplished.

In this paper, we show that preservation of the so-called spectral envelope function, a smooth (slowly varying) function of frequency which passes through most significant spectral peaks of IR training datasets, plays an important role in the design of compact and accurate SVM classifiers for qualitative IR applications. With this aim, a two-stage feature selection algorithm designed to capture main features of the spectral envelope function is presented. For this propose, a set of prospective, yet raw, spectral regions is first identified using an unsupervised approach around most significant IR peaks of the spectral envelope function. These regions are further refined using an stabilized version of the SVM-RFE algorithm with respect to variations in the training data. To favor interpretability issues, spectral regions are individually refined. In this way, core spectral envelope information gets preserved. The complete set of spectral points across refined IR regions is then used to train compact SVM classifiers.

\section{Spectral envelope functions towards effective SVM-RFE on IR data}

We notice that the problem of selecting a reduced set of discriminative wavelengths for challenging qualitative NIR applications closely resembles that of the fundamental frequency estimation of a mixture of harmonic sources in the context of music applications [30,31]. We observe that in the audio setting, data is often reduced for retaining salient information while omitting peripheral details. A strong data reduction technique of music signals is the representation of the full signal spectra to observed spectral peaks [32]. The usefulness of this approach stems from at least two facts: it is largely known that resynthesis of harmonic sounds from observed spectral peaks cause little changes in human perception [33] and for harmonic sounds, spectral peaks tend to appear at integer multiples of target fundamental frequencies. Spectral peaks define the spectral envelope. As pointed out by [34], significant peaks are required to be higher than a baseline, a kind of noise floor so that peaks under such baseline have high probabilities of being generated by noise. On the other hand, it is widely known that for quantitative IR applications, peaks of the IR spectrum are associated with characteristic vibrations of specific functional groups and thus, their heights are proportional to concentration of chemical species in samples $[35,36]$. Under these considerations, it follows that for qualitative IR applications, IR datasets may be characterized by spectral envelope functions and that these functions may be valuable for extracting potentially discriminative 
wavelengths, i.e., wavelengths associated with harmonics of core fundamental frequencies.

\subsection{Unsupervised learning of IR spectral envelope func- tions}

Let us consider a IR dataset $D$ containing $m$ training samples, each sample characterized by $n$ wavelengths, i.e., $D=\left\{d_{i}^{j}, i=1 \ldots m, j=1 \ldots n\right\}$. The raw spectral envelope function $E$ induced by $D$ (see Fig. 1-a) is given by Eq.1:

$$
E\left(x_{j}\right)=y_{j}=\max _{i \in 1 \ldots m} d_{i}^{j} \quad j \in 1 \ldots n
$$

The raw spectral envelope function $E$ is then processed for the unsupervised identification of significant peaks. Hence, all wavelengths below a baseline $b=$ median $\left(\left\{y_{j}, j=1 \ldots n\right\}\right)$ are set to $b$ (see Fig. 1-b); the choice of median rather than mean of $E$ aims to overcome the well-known sensitivity of the mean to outliers. As a result, a truncated spectral envelope function $E^{*}$ is obtained:

$$
E^{*}\left(x_{j}\right)\left\{\begin{array}{l}
y_{j} \quad y_{j}>b \quad \forall j \in 1 \ldots n \\
b \text { otherwise }
\end{array}\right.
$$

The truncated spectral envelope function $E^{*}$ is then inspected for the identification of the set $P$ of wavelengths $x_{p}$ associated with local maximums of $E^{*}$. In addition, the set $M$ of wavelengths associated with local minimums of $E^{*}$ is also computed.

\subsection{Unsupervised identification of spectral windows}

Taking into account the nature of the IR spectra, we expect that broad peaks of the truncated spectral envelope function $E^{*}$ contains important harmonics of core fundamental frequencies. Aiming to accomplish a compact representation of the IR spectra, the truncated spectral envelope function $E^{*}$ is used to guide the identification of significant spectral regions, hereafter called spectral windows. For this purpose, the Windows from Envelope (WE) algorithm (see Algorithm 1) is introduced.

Given a training IR dataset $D$, WE first computes the raw spectral envelope function $E$ (L.4), continues with a baseline $b(L .6)$ and then its truncated version $E^{*}$ with baseline $b(L .8)$. From $E^{*}$, the corresponding sets $P$ of local maximus (L.13) and the set $M$ of local minimums (L.14) are computed. For each $x_{p} \in P$, WE identifies the spectral window (L.16) centered on $x_{p}$ with width $w_{p}=$ $\left(x_{p}^{r}-x_{p}^{l}\right)$ (see Fig. 1-c), where $x_{p}^{r}$ and $x_{p}^{l}$ are respectively the right and left closer wavelengths to $x_{p}$ where $E^{*}$ falls to $\operatorname{Max}\left[b\right.$, decay $\left.* E^{*}\left(x_{p}\right)\right]$. The decay parameter, $0<$ decay $\leq 1$, is used to control spectral window widths. For sharp $E^{*}$ peaks, very narrow spectral windows are obtained despite the specific setting of the decay parameter. The resulting set of spectral windows is further processed for additional dimensionality reduction using the information about 


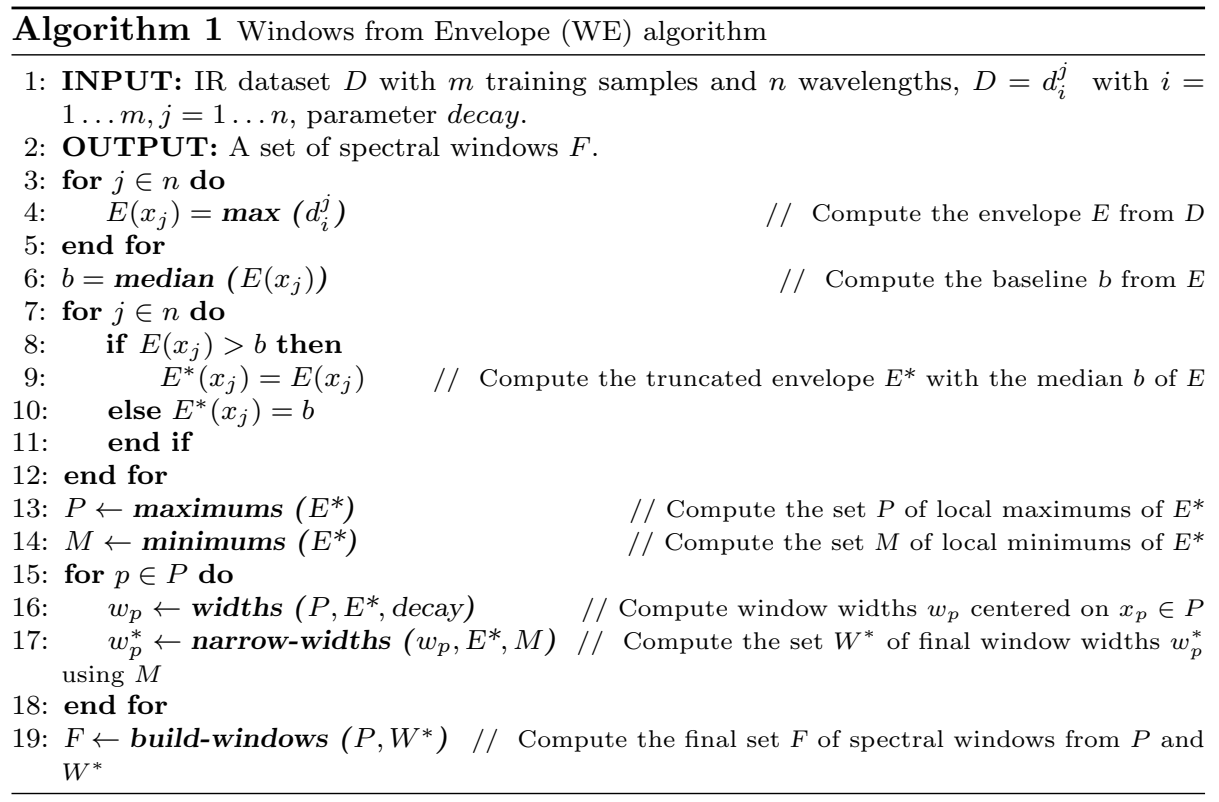

local minimums of $E^{*}$ available in $M$. Hence, narrower windows $w_{p}^{*}(L .17)$ are obtained by performing descendant walks from wavelengths $x_{p}$ until the first local minimum of $E^{*}$, if any, is found, $p=1 \ldots|P|$ (see Fig. 1-d). Afterwards, the final set of spectral windows $F(L .19)$ is built from $P$ and $W^{*}$.

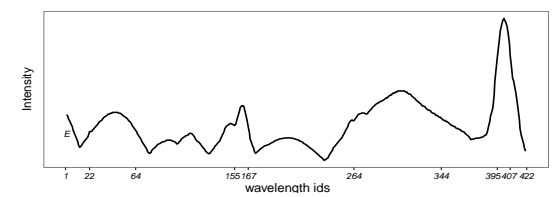

(a)

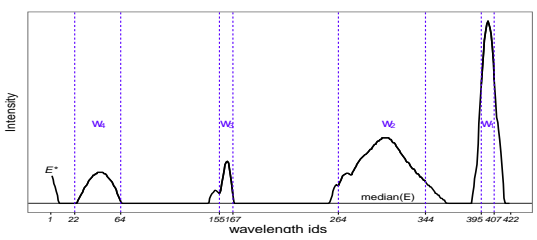

(c)

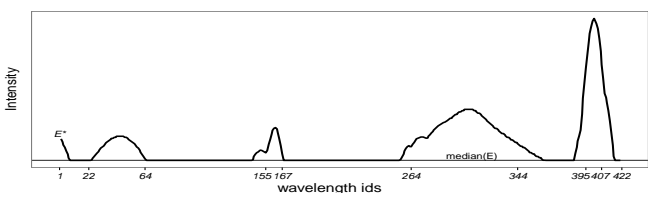

(b)

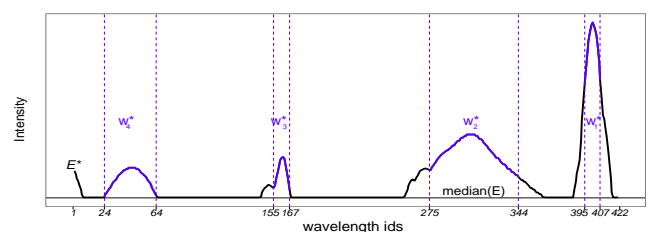

(d)

Figure 1: The unsupervised spectral envelope approach for IR data dimensionality reduction. (a) The raw spectral envelope function $E$ is induced from local maximums of IR datataset. (b) The truncated spectral envelope function $E^{*}$ is obtained with a baseline $b=\operatorname{median}\left(\left\{y_{j}, j=1 \ldots n\right\}\right)$. (c) A set of spectral windows is induced from $E^{*}$. (d) Final spectral window widths are computed using the minimums of $E^{*}$. 


\subsection{Supervised SVM-RFE refinement of spectral windows}

SVM-RFE makes feature selection using a backward elimination process based on the weights computed by a linear SVM classifier. To deal with small variations in the training data, a robust version, built upon a 5-Fold CV approach and called SVM-RFE*, was proposed by the authors [25]. To further refine the training dataset obtained after WE processing, SVM-RFE* was applied to each spectral window. The rationale behind this decision is twofold. The first reason is relate to computationally load, i.e., SVM-RFE scales quadratically with the number of features and thus, its application on a per-window basis reduces computational complexity by a factor proportional to the number of spectral windows. The second reason is related to the importance of spectral envelope functions in the characterization of IR datasets. Note that applying SVM-RFE to whole and fused set of spectral windows may drop key wavelengths for the definition of the spectral envelope function. Hence, if this function is indeed important for the characterization of IR datasets, its main features must be preserved. This objective can only be accomplished if SVM-RFE* is applied in a per-window basis mode.

Based on the above considerations, spectral windows were individually refined with an additional SVM-RFE* processing stage using a 5-Fold CV setup. Therefore, for each cross-validation run and for each SVM-RFE iteration step, a validation error was obtained using four folds for training and one fold for validation. At the end of SVM-RFE iterations, the mean validation error was computed and the smallest feature subset with a validation error below such mean was selected. Aiming to promote feature selection stability, only those features selected in the 5 cross-validation runs were finally selected. The union of feature subsets obtained for each spectral window was then used to build a reduced training dataset.

\subsection{Sensitivity analysis of SVM-RFE refinement}

In order to set the decay parameter, we analyzed its sensitivity to the combination of the WE algorithm and robust SVM-RFE (WE+SVM-RFE*) with respect to variations in the training data. To this aim, the fraction of preserved features along with their stability and the classification accuracy of corresponding linear SVM classifiers were evaluated for different settings of the decay parameter in the range $[0.5,0.9]$. Regarding the stability of feature selection, the similarity index $I_{s}$ proposed by [37] was used. Given two subsets of features $A$ and $B$, respectively obtained with decay parameters $d_{A}$ and $d_{B}$, the similarity between both subsets is given by $I_{s}=\frac{|A \cap B|}{|A \cup B|}$. To perform evaluations, a 5-Fold CV approach on the two following IR datasets was considered:

Diesel: This dataset, obtained from data in [38], contains 60 NIR samples of three types of gasoline (17, 23 and 20 samples) defined by their octane number. Each NIR sample consists of 401 wavelengths in the range of 900-1700 nm. 
Wine: This dataset, provided by Marc Meurens ${ }^{1}$, contains 124 MIR samples of three types of wine (37, 36 and 48 samples) defined by their alcohol level. Each MIR sample consists of 252 wavelengths in the range of $400-4000 \mathrm{~cm}^{-1}$.

Average 5-Fold CV results on the two datasets for the fraction of selected features (see Fig. 2a) and the classification accuracy of corresponding SVM classifiers (see Fig. 2b) suggested that a decay parameter between 0.65 and 0.8 may lead to satisfactory performance results. To make a final decision on a robust value for the decay parameter, feature selection stability results (see Tables 1 and 2) were analyzed. Hence, we searched for decay pairs in the grid $[0.5,0.9] \times[0.5,0.9]$ showing the highest $I_{s}$ values with the smallest variations near the diagonals. As a result of this analysis, even if other values are also possible, the decay parameter was set to 0.75 .

Table 1: WE+SVM-RFE* feature selection stability on the Diesel dataset for different settings of the decay parameter. Average 5-Fold $\mathrm{CV}$ values of the Kalousis index $I_{s}$ are reported for decay parameter pairs $\left(d_{a}, d_{b}\right)$ on the grid $[0.5,0.55, \ldots, 0.9] \times[0.5,0.55, \ldots, 0.9]$.

\begin{tabular}{c|ccccccccc}
\hline$d_{a} \backslash d_{b}$ & 0.50 & 0.55 & 0.60 & 0.65 & 0.70 & 0.75 & 0.80 & 0.85 & 0.90 \\
\hline 0.50 & 1.00 & 0.45 & 0.44 & 0.41 & 0.42 & 0.44 & 0.36 & 0.37 & 0.31 \\
0.55 & 0.45 & 1.00 & 0.49 & 0.45 & 0.42 & 0.41 & 0.34 & 0.34 & 0.32 \\
0.60 & 0.44 & 0.49 & 1.00 & 0.77 & 0.69 & 0.63 & 0.52 & 0.56 & 0.42 \\
0.65 & 0.41 & 0.45 & 0.77 & 1.00 & 0.78 & 0.69 & 0.58 & 0.57 & 0.48 \\
0.70 & 0.42 & 0.42 & 0.69 & 0.78 & 1.00 & 0.82 & 0.65 & 0.63 & 0.52 \\
0.75 & 0.44 & 0.41 & 0.63 & 0.69 & 0.82 & 1.00 & 0.79 & 0.63 & 0.50 \\
0.80 & 0.36 & 0.34 & 0.52 & 0.58 & 0.65 & 0.79 & 1.00 & 0.65 & 0.47 \\
0.85 & 0.37 & 0.34 & 0.56 & 0.57 & 0.63 & 0.63 & 0.65 & 1.00 & 0.62 \\
0.90 & 0.31 & 0.32 & 0.42 & 0.48 & 0.52 & 0.50 & 0.47 & 0.62 & 1.00 \\
\hline
\end{tabular}

Table 2: WE+SVM-RFE* feature selection stability on the Wine dataset for different settings of the decay parameter. Average 5 -Fold $\mathrm{CV}$ values of the Kalousis index $I_{s}$ are reported for decay parameter pairs $\left(d_{a}, d_{b}\right)$ on the grid $[0.5,0.55, \ldots, 0.9] \times[0.5,0.55, \ldots, 0.9]$.

\begin{tabular}{c|ccccccccc}
\hline$d_{a} \backslash d_{b}$ & 0.50 & 0.55 & 0.60 & 0.65 & 0.70 & 0.75 & 0.80 & 0.85 & 0.90 \\
\hline 0.50 & 1.00 & 0.49 & 0.49 & 0.43 & 0.39 & 0.35 & 0.26 & 0.19 & 0.14 \\
0.55 & 0.49 & 1.00 & 0.41 & 0.41 & 0.36 & 0.31 & 0.22 & 0.18 & 0.11 \\
0.60 & 0.49 & 0.41 & 1.00 & 0.67 & 0.41 & 0.51 & 0.40 & 0.23 & 0.12 \\
0.65 & 0.43 & 0.41 & 0.67 & 1.00 & 0.50 & 0.68 & 0.40 & 0.27 & 0.11 \\
0.70 & 0.39 & 0.36 & 0.41 & 0.50 & 1.00 & 0.63 & 0.41 & 0.35 & 0.14 \\
0.75 & 0.35 & 0.31 & 0.51 & 0.68 & 0.63 & 1.00 & 0.65 & 0.37 & 0.12 \\
0.80 & 0.26 & 0.22 & 0.40 & 0.40 & 0.41 & 0.65 & 1.00 & 0.51 & 0.21 \\
0.85 & 0.19 & 0.18 & 0.23 & 0.27 & 0.35 & 0.37 & 0.51 & 1.00 & 0.37 \\
0.90 & 0.14 & 0.11 & 0.12 & 0.11 & 0.14 & 0.12 & 0.21 & 0.37 & 1.00 \\
\hline
\end{tabular}

\footnotetext{
${ }^{1}$ http://mlg.info.ucl.ac.be/index.php?page=DataBases
} 

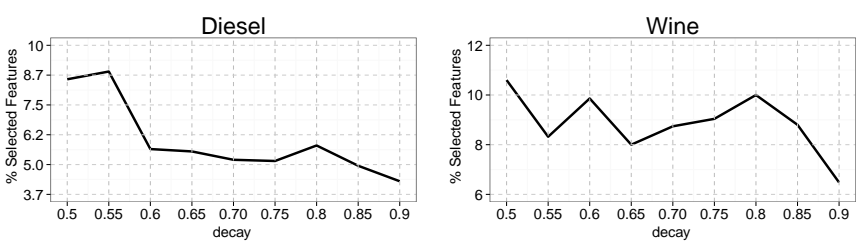

(a)
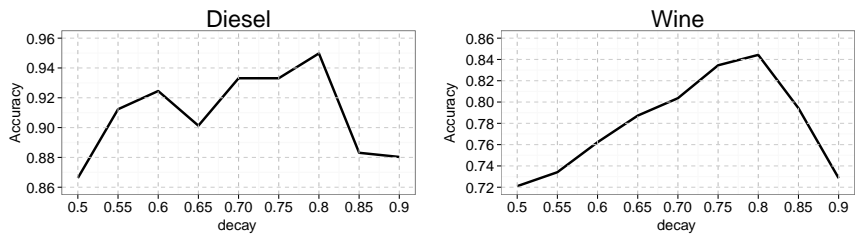

(b)

Figure 2: (a) The fraction of selected features by the WE+SVM-RFE* method on the Diesel and Wine datasets for different settings of the decay parameter. Average 5-Fold CV values are reported for decay in the range $[0.5,0.55, \ldots, 0.9]$. (b) SVM classification accuracy on the Diesel and Wine datasets. Average 5-Fold CV precision values are reported when WE+SVM$\mathrm{RFE}^{*}$ feature selection is performed with the decay parameter in the range $[0.5,0.55, \ldots, 0.9]$.

\section{$3 \quad$ Numerical experiments}

\subsection{Description of used datasets}

Multiple datasets across different IR domains were selected for evaluating the performance of the WE+SVM-RFE* feature selection algorithm in the construction of accurate and interpretable linear SVM classifiers.

Polymers: This dataset was given from the XXX Project with $\mathrm{XXX}^{2}$ contains NIR samples of four types of plastic bottles, namely, PET (47 samples), PEHD (125 samples), Polypropylene (50 samples) and PVC (89 samples). In order to be self-contained, a brief description of sample collection is made. NIR samples were obtained using a reflexion setup with a halogen light source set to irradiate plastic bottles and a white screen behind them to reflect the light. NIR spectra were acquired using a StellarNet spectrometer (950 to $1700 \mathrm{~nm}$, Black comet model, 256 pixels) controlled by a computer via USB. The wavelength region was chosen because it contains several plastic absorption bands. Bottles were placed with the head below on a moving metallic stick and measurements were performed on the bottom of the bottle in order to reduce interference and be sure no liquid remained, which would dramatically affect spectral signatures. NIR measurements were performed at $2 \mathrm{~nm}$ intervals thus giving 422 wavelengths per sample.

Apricots. This dataset, derived from [39], contains 731 MIR samples of apricots of three types (230, 244 and 257 samples) defined by their Brix degree,

${ }^{2}$ http://www.ondalys.fr/ 
i.e., by their water-soluble sugar concentration. MIR samples consist of 292 wavelengths in the range of $900-1500 \mathrm{~cm}^{-1}$.

Strawberry. This dataset ${ }^{3}$ contains 983 MIR samples of two types of fruit pures, namely "Strawberry" (632 samples) and "Non-Strawberry" (251 samples) [40]. In the former case, pures are prepared from fresh whole fruits by the researchers. In the latter case, purées are prepared from diverse collection of other purées, including strawberry adulterated with other fruits and sugar solutions, raspberry, apple, blackcurrant, blackberry, plum, cherry, apricot, grape juice and mixtures of these. MIR samples consisting of 235 wavelengths in the range $899-1802 \mathrm{~cm}^{-1}$ were acquired from each purée sample using attenuated total reflectance sampling.

\subsection{Experimental protocol}

The effectiveness of the WE+SVM-RFE* feature selection method in the construction of accurate and compact SVM classifiers for IR data was compared

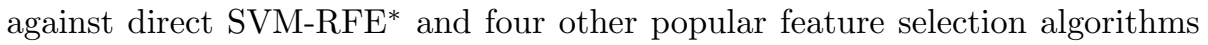
mentioned in the literature. Specifically, we first considered the SVM-RFE* approach that eliminates the least useful feature at each iteration step. We also considered Relief [41], a well-known feature subset selection algorithm known to handle strong dependencies between features and noise, and three entropybased feature selection algorithms [42]: Information Gain (InfoGain), Information Gain Ratio (GainR) and Symmetrical Uncertainty (SymmU), all of them assuming independence between features. One of the three methods of entropybased feature selection, InfoGain, was evaluated.

For the sake of completeness, dimensionality reduction methods were also considered. These methods involve a space transformation which makes hard the interpretation using the initial, raw, features. Nevertheless, they are widely used as they do not require a feature selection process and they are able to exploit the whole information of the input spectra. Principal Component Analysis (PCA), Linear Discriminant Analysis (LDA) and Partial Least Squares Discriminant Analysis (PLS-DA) methods share the way the new space is defined: the axes are linear combinations of the raw features. They differ in the way these axes are designed. PCA [43] maximizes the explained variance of the input spectra, the axes are the eigenvectors of $X^{T} X$. To make a classification, SVM classifiers are evaluated in the new space. LDA [44] maximizes the between group variance, $B$. The axes are the eigenvectors of $T^{-1} B$, where $T$ stands for the total variance matrix. Finally, PLS-DA [45], maximizes the covariance between the input spectra and the target. The axes are computed using iterative algorithms.

A randomized strategy based on $50 \times 5$-Fold CV experiments was used to assess the performance of aforementioned feature selection and dimensionality reduction methods. At each CV fold, an inner 5-Fold CV experiment was performed to estimate the optimal number of features in the SVM-RFE, Relief, InfoGain feature selection methods and the optimal number of components in

${ }^{3}$ http://www.ifr.ac.uk/Bioinformatics/MIRFruitPurees.zip 
Table 3: The number of features selected by WE+SVM-RFE*, WE+SVM-RFE* and SVM-RFE* feature selection methods along with the classification accuracy accomplished by corresponding linear SVM classifiers in a 5-Fold CV steup.

\begin{tabular}{lccccc}
\hline Dataset & Type & \# Features & WE+SVM-RFE* & WE+SVM-RFE & SVM-RFE* $^{*}$ \\
\hline Diesel & NIR & 401 & $16(0.94)$ & $13(0.80)$ & $14(0.80)$ \\
Wine & MIR & 252 & $18(0.82)$ & $19(0.78)$ & $18(0.79)$ \\
\hline
\end{tabular}

the PCA dimensionality reduction technique. Feature selection performance was evaluated by the mean number of features selected across the 50 runs of 5-Fold CV experiments. Similarly, linear SVM classifiers built after feature selection, PCA dimensionality reduction, PLS-DA and LDA classifiers were evaluated with the mean classification accuracy.

In practice, the default implementations of the Relief and entropy-based feature selection methods provided in the R package "FSelector" [46] were used for supervised feature selection. Similarly, the prcomp implementation of PCA algorithm provided in the $\mathrm{R}$ Stats package [47] was used for unsupervised dimensionality reduction. Finally, the R package "plsgenomics" [48] was used for optimized LDA classification and "mixOmics" [49] was used for PLS-DA classification.

\section{Results and discussion}

\subsection{The importance of the spectral envelope function}

To appreciate the importance of the spectral envelope function in the characterization of IR datasets, three operation modes of SVM-RFE* were evaluated: i) per-spectral window after WE processing ( $\left.\left.\mathrm{WE}+\mathrm{SVM}-\mathrm{RFE}^{*}\right), i i\right)$ all spectral windows after WE processing (WE+SVM-RFE ${ }_{a}^{*}$ ), and $\left.i i i\right)$ the complete set of wavelengths in the original training data.

A 5-Fold CV approach on Diesel and Wine datasets was considered. Average 5-Fold CV results for the number of selected features and the classification accuracy (see Table 3) of corresponding linear SVM classifiers suggest that using SVM-RFE* in the per-window operation mode is the best data processing strategy and that there is no advantage in applying SVM-RFE* to all spectral windows over all wavelengths in the original training data.

We wonder whether these results may be due to the preservation of wavelengths of the spectral envelope function accomplished by the SVM-RFE* algorithm when used in the per-spectral window operation mode. To shed some light on this issue, selected features in three SVM-RFE* operation modes were mapped to reference spectral windows obtained after WE processing. It was observed that wavelengths of the spectral envelope functions were practically dismissed by SVM-RFE* when used in the all-wavelengths operation mode and, were only partially preserved in the all-spectral windows operation mode (see Tables 4 and 5). Overall, these results suggest that the preservation of main wavelengths of the spectral envelope function accomplished by the WE+SVM- 
Table 4: WE+SVM-RFE*, WE+SVM-RFE* and SVM-RFE* feature selection in the Diesel dataset. Selected wavelengths (ID numbers) are mapped against reference WE spectral windows specified by their lower and upper wavelength limits.

\begin{tabular}{|c|c|c|c|}
\hline \multirow[b]{2}{*}{ Feature selection } & \multicolumn{3}{|c|}{ WE spectral windows } \\
\hline & {$[122-128]$} & {$[144-150]$} & {$[239-255]$} \\
\hline WE+SVM-RFE* & $\{122-125,128\}$ & $\{146-147,149-150\}$ & $\{245-247,249-252\}$ \\
\hline $\mathrm{WE}+\mathrm{SVM}^{-\mathrm{RFE}_{g}^{*}}$ & $\{124-125,127\}$ & $\{145-146,148\}$ & $\{245-251\}$ \\
\hline SVM-RFE & & & $\{239,251\}$ \\
\hline
\end{tabular}

Table 5: WE+SVM-RFE*, WE+SVM-RFE* and SVM-RFE* feature selection in the Wine dataset. Selected wavelengths (ID numbers) are mapped against reference WE spectral windows specified by their lower and upper wavelength limits

\begin{tabular}{lcccccc}
\hline & \multicolumn{5}{c}{ WE spectral windows } \\
\cline { 2 - 7 } Feature selection & {$[24-28]$} & {$[33-37]$} & {$[83-90]$} & {$[93-108]$} & {$[117-126]$} & {$[129-133]$} \\
\hline WE+SVM-RFE* & $\{25,27\}$ & $\{34\}$ & $\{85,88-90\}$ & $\{93-94,104,106,108\}$ & $\{117,120,126\}$ & $\{130\}$ \\
WE+SVM-RFE $_{g}^{*}$ & & & $\{84,87-88\}$ & $\{96-100\}$ & $\{117-120\}$ & $\{129-130,133\}$ \\
SVM-RFE* $^{2}$ & $\{24-28\}$ & & & & &
\end{tabular}

$\mathrm{RFE}^{*}$ algorithm is an important issue for the construction of compact and accurate linear SVM classifiers for IR datasets.

\subsection{WE+SVM-RFE* performance}

To better understand the difficulty of the three classification problems at hand, a $2 \mathrm{D}$ visualization analysis was first performed using PCA. Highly overlapped classes, with no clear linear separation boundaries were observed in all cases. These results suggested the need of dimensionality reduction, e.g., by means of PCA, or feature selection before any classification algorithm could be applied.

Regarding interpretability, Table 6 shows firstly that WE+SVM-RFE* leads to the smallest sets of features compared to other alternatives, including SVMRFE*$^{*}$. Further screening showed that selected features with WE+SVM-RFE*, as opposed to SVM-RFE*, tend to be always contained in a reduced number of spectral regions associated with more salient peaks, which seem to be related to target classes. For instance, in the Polymer dataset the four spectral regions (A, B, C, D) selected by WE+SVM-RFE* method (see Fig. 3) point out main features of the four plastic bottle spectrum (PVC, PET, PEHD and polypropylene) [50]. On the other hand, features selected by the raw SVM-RFE* are dispersed across the full spectrum (lines in grey), which makes difficult the interpretation. Altogether, these results suggest the usefulness of the proposed method when both interpretability and classification of IR spectrum are of interest.

Regarding accuracy, Table 7 shows that WE+SVM-RFE* yields similar, or even better, results than the concurrent approaches, including the optimized LDA, PLS-DA and PCA based SVM classifiers. The largest gain is for the Apricot dataset. 
Table 6: The number of features selected by the WE+SVM-RFE*, SVM-RFE*, InfoGain, GainR, SymmU and Relief feature selection methods on benchmark NIR/MIR datasets. In the fourth column between brackets the number of selected regions of the spectrum is expressed.

\begin{tabular}{lcccccc}
\hline Dataset & Type & \# Features & WE+SVM-RFE*(\#Regions)SVM-RFE* & InfoGain & Relief \\
\hline Polymer & NIR & 422 & $62(4)$ & 90 & 97 & 80 \\
Apricot & MIR & 292 & $20(4)$ & 35 & 78 & 32 \\
Strawberry MIR & 235 & $45(6)$ & 70 & 188 & 106 \\
\hline
\end{tabular}

Table 7: The classification accuracy accomplished by linear SVM classifiers after the application of the WE+SVM-RFE*, SVM-RFE*, InfoGain and Relief feature selection methods and the PCA dimensionality reduction technique to benchmark NIR/MIR datasets. The classification accuracy of optimized LDA and PLS-DA classifiers are shown as reference.

\begin{tabular}{lcccccc|cc}
\hline Dataset & T ype & \multicolumn{5}{c|}{ SVM } & & \\
& & WE+SVM-RFE* & SVM-RFE* & InfoGain & Relief & PCA & LDA & PLS-DA \\
\hline Polymer & NIR & 0.95 & 0.93 & 0.95 & 0.92 & 0.95 & 0.93 & 0.95 \\
Apricot & MIR & 0.96 & 0.85 & 0.87 & 0.85 & 0.90 & 0.85 & 0.91 \\
Strawberry & MIR & 0.98 & 0.90 & 0.98 & 0.96 & 0.96 & 0.96 & 0.97 \\
\hline
\end{tabular}

\section{Conclusions}

In this paper, a spectral envelope approach towards effective SVM-RFE on IR datasets has been presented. As it happens with music applications, the spectral envelope function provides a high level and compact representation of IR datasets and thus, subject to suitable processing, it may be used to overcome the difficulties found in the direct application of the SVM-RFE method. These considerations motivate the introduction of the Windows from Envelope algorithm allowing the unsupervised identification of a reduced set of spectral windows supporting the spectral envelope function and thus, the effective application of the SVM-RFE method on IR datasets. Taking into account the well-known sensitivity of SVM classifiers to noise and outliers [14] and that a variety of noise sources may affect the quality of IR datasets [51], an ensemble approach to SVM-RFE was used.

These insights are captured in the WE+SVM-RFE* proposal for feature selection on IR datasets. Experimental results across three different IR application domains (polymers, agriculture and food) demonstrated that spectral regions achieved with WE+SVM-RFE* can shed light on the relation between spectral regions and target classes. Finally, experimental results across three different IR application domains (polymers, agriculture, and food) suggest the usefulness of the proposed method for the construction of compact, interpretable and accurate SVM classification models for qualitative IR applications.

\section{Acknowledgments}

The authors were supported by projects PICT PRH No. 0253 (2011) and No. 2513 (2012), ANPCyT, Argentina. 


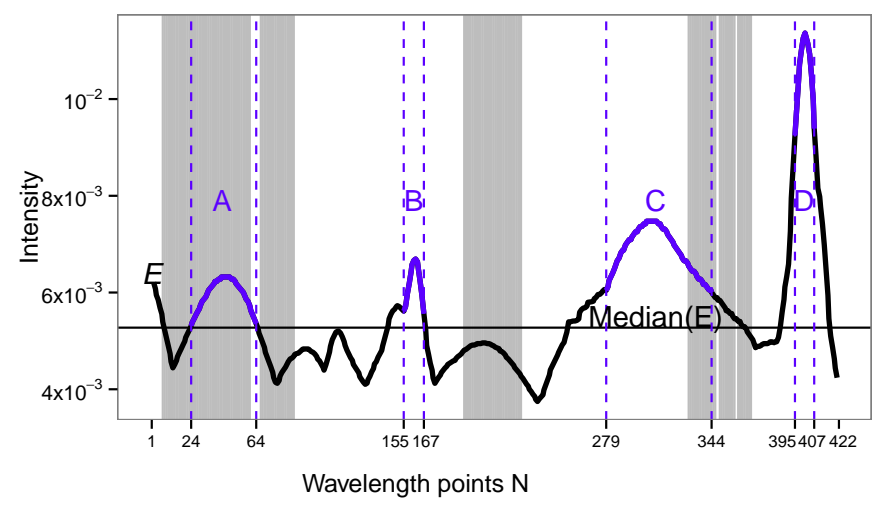

Figure 3: Polymer envelope function. Dashed blue lines represent the selected regions with WE+SVM-RFE*. Grey lines represent the selected features with SVM-RFE*.

\section{References}

[1] Y. Ge, J. Thomasson, R. Sui, Remote sensing of soil properties in precision agriculture: A review, Frontiers of Earth Science 5 (3) (2011) 229-238.

[2] R. V. Rossel, D. Walvoort, A. McBratney, L. Janik, J. Skjemstad, Visible, near infrared, mid infrared or combined diffuse reflectance spectroscopy for simultaneous assessment of various soil properties, Geoderma 131 (12) (2006) 59-75.

[3] D. Ferreira, J. Pallone, R. Poppi, Direct analysis of the main chemical constituents in chenopodium quinoa grain using fourier transform nearinfrared spectroscopy, Food Control 48 (0) (2015) 41-45.

[4] A. Fudge, K. L. Wilkinson, R. Ristic, D. Cozzolino, Classification of smoke tainted wines using mid-infrared spectroscopy and chemometrics, J Agric Food Chem.

[5] X. Li, Y. He, H. Fang, Non-destructive discrimination of chinese bayberry varieties using vis/nir spectroscopy, Journal of Food Engineering 81 (2) (2007) 357-363.

[6] D. M. Beckles, Factors affecting the postharvest soluble solids and sugar content of tomato (solanum lycopersicum l.) fruit, Postharvest Biology and Technology 63 (1) (2012) 129 - 140.

[7] B. M. Nicola, K. Beullens, E. Bobelyn, A. Peirs, W. Saeys, K. I. Theron, J. Lammertyn, Nondestructive measurement of fruit and vegetable quality by means of $\{$ NIR $\}$ spectroscopy: A review, Postharvest Biology and Technology 46 (2) (2007) 99-118. 
[8] A. Kassouf, J. Maalouly, D. N. Rutledge, H. Chebib, V. Ducruet, Rapid discrimination of plastic packaging materials using $\{\mathrm{MIR}\}$ spectroscopy coupled with independent components analysis (ica), Waste Management 34 (11) (2014) 2131-2138.

[9] T. Mehmood, K. H. Liland, L. Snipen, S. Sb, A review of variable selection methods in partial least squares regression, Chemometrics and Intelligent Laboratory Systems 118 (0) (2012) $62-69$.

[10] Y. Langeron, M. Doussot, D. Hewson, J. Duchne, Classifying nir spectra of textile products with kernel methods, Engineering Applications of Artificial Intelligence 20 (3) (2007) $415-427$.

[11] A.-L. Boulesteix, K. Strimmer, Partial least squares: a versatile tool for the analysis of high-dimensional genomic data, Briefings in Bioinformatics 8 (1) (2007) 32-44.

[12] G. Gurdeniz, B. Ozen, Detection of adulteration of extra-virgin olive oil by chemometric analysis of mid-infrared spectral data, Food Chemistry 116 (2) (2009) 519-525.

[13] W. Suphamitmongkol, G. Nie, R. Liu, S. Kasemsumran, Y. Shi, An alternative approach for the classification of orange varieties based on near infrared spectroscopy, Computers and Electronics in Agriculture 91 (0) (2013) 87-93.

[14] B. E. Boser, I. M. Guyon, V. N. Vapnik, A training algorithm for optimal margin classifiers, in: Proceedings of the Fifth Annual Workshop on Computational Learning Theory, COLT '92, ACM, New York, NY, USA, 1992, pp. $144-152$.

[15] V. Vapnik, Universal learning technology: Support vector machines, NEC Journal of Advanced Technology 2 (2) (2005) 137-144.

[16] S. Ramaswamy, P. Tamayo, Rifkin, et al, Multiclass cancer diagnosis using tumor gene expression signatures., Proceedings of the National Academy of Sciences of the United States of America 98 (26) (2001) 15149-15154.

[17] G. Guo, S. Z. Li, Content-based audio classification and retrieval by support vector machines, IEEE Transactions on Neural Networks 14 (1) (2003) 209215.

[18] Y. Xu, S. Zomer, R. G. Brereton, Support vector machines: A recent method for classification in chemometrics, Critical Reviews in Analytical Chemistry 36 (3-4) (2006) 177-188.

[19] Q. Chen, J. Zhao, C. Fang, D. Wang, Feasibility study on identification of green, black and oolong teas using near-infrared reflectance spectroscopy based on support vector machine (svm), Spectrochimica Acta Part A: Molecular and Biomolecular Spectroscopy 66 (3) (2007) 568-574. 
[20] O. Devos, C. Ruckebusch, A. Durand, L. Duponchel, J.-P. Huvenne, Support vector machines (svm) in near infrared (nir) spectroscopy: Focus on parameters optimization and model interpretation, Chemometrics and Intelligent Laboratory Systems 96 (1) (2009) 27 - 33.

[21] Y. Saeys, I. Inza, P. Larraaga, A review of feature selection techniques in bioinformatics, Bioinformatics 23 (19) (2007) 2507-2517.

[22] I. Guyon, J. Weston, S. Barnhill, V. Vapnik, Gene selection for cancer classification using support vector machines, Machine Learning 46 (1-3) (2002) 389-422.

[23] S. Deng, Y. Xu, L. Li, X. Li, Y. He, A feature-selection algorithm based on support vector machine-multiclass for hyperspectral visible spectral analysis, Journal of Food Engineering 119 (1) (2013) 159 - 166.

[24] A. Kalousis, J. Prados, M. Hilario, Stability of feature selection algorithms: A study on high-dimensional spaces, Knowl. Inf. Syst. 12 (1) (2007) 95-116.

[25] E. Tapia, P. Bulacio, L. Angelone, Sparse and stable gene selection with consensus svm-rfe, Pattern Recognition Letters 33 (2) (2012) 164-172.

[26] P. Mundra, J. Rajapakse, Svm-rfe with mrmr filter for gene selection, NanoBioscience, IEEE Transactions on 9 (1) (2010) 31-37.

[27] M. Ghasemi-Varnamkhasti, M. Forina, \{NIR $\}$ spectroscopy coupled with multivariate computational tools for qualitative characterization of the aging of beer, Computers and Electronics in Agriculture 100 (0) (2014) 34 40.

[28] R. Moscetti, R. P. Haff, S. Saranwong, D. Monarca, M. Cecchini, R. Massantini, Nondestructive detection of insect infested chestnuts based on \{NIR\} spectroscopy, Postharvest Biology and Technology 87 (0) (2014) $88-94$.

[29] Q. Yuhua, D. Xiangqian, G. Huili, Application of high-dimensional feature selection in near-infrared spectroscopy of cigarettes' qualitative evaluation, Spectroscopy Letters 46 (6) (2013) 397-402.

[30] G. E. Poliner, D. Ellis, A. Ehmann, E. Gomez, S. Streich, B. Ong, Melody transcription from music audio: Approaches and evaluation, Audio, Speech, and Language Processing, IEEE Transactions on 15 (4) (2007) 1247-1256.

[31] M. Casey, R. Veltkamp, M. Goto, M. Leman, C. Rhodes, M. Slaney, Content-based music information retrieval: Current directions and future challenges, Proceedings of the IEEE 96 (4) (2008) 668-696.

[32] Z. Duan, B. Pardo, C. Zhang, Multiple fundamental frequency estimation by modeling spectral peaks and non-peak regions, Audio, Speech, and Language Processing, IEEE Transactions on 18 (8) (2010) 2121-2133. 
[33] J. O. Smith, X. Serra, PARSHL: An analysis/synthesis program for nonharmonic sounds based on a sinusoidal representation, in: International Computer Music Conference (ICMC), International Computer Music Association, 1987, pp. 290-297.

[34] Z. Duan, Y. Zhang, C. Zhang, Z. Shi, Unsupervised single-channel music source separation by average harmonic structure modeling, Audio, Speech, and Language Processing, IEEE Transactions on 16 (4) (2008) 766-778.

[35] B. Smith, Infrared Spectral Interpretation, CRC Press, 1998.

[36] B. H. Stuart, Infrared Spectroscopy: Fundamentals and Applications, John Wiley \& Sons, Ltd, 2005.

[37] A. Kalousis, J. Prados, M. Hilario, Stability of feature selection algorithms, in: Data Mining, Fifth IEEE International Conference on, 2005, p. 8.

[38] J. H. Kalivas, Two data sets of near infrared spectra, Chemometrics and Intelligent Laboratory Systems 37 (2) (1997) 255-259.

[39] S. Bureau, D. Ruiz, M. Reich, B. Gouble, D. Bertrand, J.-M. Audergon, C. M. Renard, Rapid and non-destructive analysis of apricot fruit quality using ft-near-infrared spectroscopy, Food Chemistry 113 (4) (2009) 13231328.

[40] E. K. Holland, J. K .and Kemsley, R. H. Wilson, Use of fourier transform infrared spectroscopy and partial least squares regression for the detection of adulteration of strawberry pures, Journal of the Science of Food and Agriculture 76 (2) (1998) 263-269.

[41] K. Kira, L. A. Rendell, A practical approach to feature selection, in: Proceedings of the Ninth International Workshop on Machine Learning, ML92, Morgan Kaufmann Publishers Inc., San Francisco, CA, USA, 1992, pp. 249-256.

[42] T. M. Mitchell, Machine Learning, 1st Edition, McGraw-Hill, Inc., New York, NY, USA, 1997.

[43] I. T. Jolliffe, Principal Component Analysis, 2nd Edition, Springer, 2002.

[44] A. L. B. Brito, L. R. Brito, F. A. Honorato, M. J. C. Pontes, L. F. B. L. Pontes, Classification of cereal bars using near infrared spectroscopy and linear discriminant analysis, Food Research International 51 (2) (2013) 924928.

[45] M. Barker, W. Rayens, Partial least squares for discrimination, Journal of Chemometrics 17 (3) (2003) 166-173.

[46] P. Romanski, FSelector, r package version 3.1.0 (2014).

[47] R-Core-Team, The R Stats Package, r package version 3.1.0 (2014). 
[48] A. L. Boulesteix, S. L. Lacroix, J. Peyre, K. Strimmer, plsgenomics, r package version 3.1 .0 (2015).

[49] K.-A. L. Cao, I. Gonzalez, S. Dejean, mixOmics, r package version 3.1.0 (2015).

[50] U. Cambridge, Ir spectra for some common polymers, accessed: 2015-10-29 (2015).

URL http://www.doitpoms.ac.uk/tlplib/artefact/polymers.php

[51] L. Xu, Y.-P. Zhou, L.-J. Tang, H.-L. Wu, J.-H. Jiang, G.-L. Shen, R.-Q. $\mathrm{Yu}$, Ensemble preprocessing of near-infrared (nir) spectra for multivariate calibration, Analytica Chimica Acta 616 (2) (2008) 138-143. 